\title{
Effect of Watch-Type Haptic Metronome on the Quality of Cardiopulmonary Resuscitation: A Simulation Study
}

\author{
Boram Choi ${ }^{1}$, Taerim Kim ${ }^{1}$, Sun Young Yoon ${ }^{1}$, Jun Sang Yoo ${ }^{2}$, Ho-Jeong Won ${ }^{3}$, Kyunga Kim ${ }^{3}$, \\ Eun Jin Kang ${ }^{1}$, Hee Yoon $^{1}$, Sung Yeon Hwang ${ }^{1}$, Tae Gun Shin ${ }^{1}$, Min Seob Sim ${ }^{1}$, Won Chul Cha ${ }^{1,2}$ \\ 'Department of Emergency Medicine, Samsung Medical Center, Sungkyunkwan University School of Medicine, Seoul, Korea \\ ${ }^{2}$ Department of Digital Health, Samsung Advanced Institute for Health Sciences and Technology, Sungkyunkwan University, Seoul, Korea \\ ${ }^{3}$ Biostatistics and Clinical Epidemiology Center, Samsung Medical Center, Seoul, Korea
}

Objectives: The aim of this study was to test the applicability of haptic feedback using a smartwatch to the delivery of cardiac compression (CC) by professional healthcare providers. Methods: A prospective, randomized, controlled, case-crossover, standardized simulation study of 20 medical professionals was conducted. The participants were randomly assigned into haptic-first and non-haptic-first groups. The primary outcome was an adequate rate of $100-120 / \mathrm{min}$ of CC. The secondary outcome was a comparison of CC rate and adequate duration between the good and bad performance groups. Results: The mean interval between CCs and the number of haptic and non-haptic feedback-assisted CCs with an adequate duration were insignificant. In the subgroup analysis, both the good and bad performance groups showed a significant difference in the mean CC interval between the haptic and non-haptic feedback-assisted CC groups-good: haptic feedback-assisted $(0.57-0.06)$ vs. non-haptic feedback-assisted (0.54-0.03), $p<0.001$; bad: haptic feedback-assisted (0.57-0.07) vs. non-haptic feedback-assisted (0.58-0.18), $p=0.005$ - and the adequate chest compression number showed significant differencesgood: haptic feedback-assisted (1,597/75.1\%) vs. non-haptic feedback-assisted (1,951/92.2\%), $p<0.001$; bad: haptic feedbackassisted $(1,341 / 63.5 \%)$ vs. non-haptic feedback-assisted (523/25.4\%), $p<0.001$. Conclusions: A smartwatch cardiopulmonary resuscitation feedback system could not improve rescuers' CC rate. According to our subgroup analysis, participants might be aided by the device to increase the percentage of adequate compressions after one minute.

Keywords: Smartphone, Cardiopulmonary Resuscitation, Heart Massage, Feedback, Simulation Training

Submitted: July 13, 2019

Revised: September 15, 2019

Accepted: October 18, 2019

\section{Corresponding Author}

Won Chul Cha

Department of Digital Health, Samsung Advanced Institute for Health Sciences and Technology, Sungkyunkwan University, 81 Irwon-ro, Gangnam-gu, Seoul 06351, Korea. Tel: +82-2-3410-2053, E-mail: wc.cha@samsung.com (https://orcid.org/0000-0002-27782992)

This is an Open Access article distributed under the terms of the Creative Commons Attribution Non-Commercial License (http://creativecommons.org/licenses/by$\mathrm{nc} / 4.0 /$ ) which permits unrestricted non-commercial use, distribution, and reproduction in any medium, provided the original work is properly cited.

(C) 2019 The Korean Society of Medical Informatics

\section{Introduction}

The survival rate from cardiac arrest remains poor despite ongoing efforts to improve the 'chain of survival' over the past 50 years [1-4]. The delivery of high-quality cardiopulmonary resuscitation (CPR), while ensuring chest compression of adequate rate and depth, and allowing full chest recoil, was found to provide a significant survival benefit in previous studies [5,6]. Although the American Heart Association (AHA) and European Resuscitation Council (ERC) guidelines clearly define how to achieve effective CPR in basic life support (BLS) and advanced cardiac life support (ACLS) [7-9], maintaining good quality of CC in the field 
has been reported to be challenging [10].

Therefore, various types of feedback or prompt devices have been introduced to improve the quality of cardiac compression (CC) [11-14]. The most updated AHA and ERC guidelines recommend using audiovisual feedback devices during CPR training to improve skill acquisition and retention $[15,16]$. In the review of interventions to improve CPR quality, not only audiovisual feedback devices but also mobile phones with BLS software or applications also showed favorable outcomes in improving the quality of bystander CPR in the prehospital setting [17]. However, these feedback devices are not as effective as expected in crowded and noisy environments, especially in the hospital setting [18].

Recently, a feedback system using a smartwatch was introduced as a substitute [19-21]. Haptic feedback using vibrations from a smartwatch can be more effectively applicable than an audiovisual feedback device in the crowded and noisy environments of emergency settings [22]. In addition, smartwatches can give feedback according to each rescuer and can be worn without disturbing the rescuer's movement.

Although previous studies have demonstrated smartwatches as potential feedback devices for high-quality CC, the outcomes are diverse in terms of CPR quality [19-21].
Furthermore, most of these studies have used either visual or audiovisual feedback in laypersons or medical students. Our study aimed to test the effect of haptic feedback using a smartwatch in the delivery of CC by professional healthcare providers. We hypothesized that haptic feedback using a smartwatch would increase the quality of CPR performed by professional healthcare providers.

\section{Methods}

\section{Study Design}

We designed a prospective, randomized, controlled, standardized simulation study to determine the effect of a haptic device on CPR quality. The study was approved by the Institutional Review Board of Samsung Medical Center (No. 2018-12-014-001).

\section{Study Participants}

Twenty participants were recruited from the study site by posting recruitment information on the employee notice board. Medical professionals who were either BLS or ACLS certified were eligible for study participation. Informed consent was obtained from all the participants.

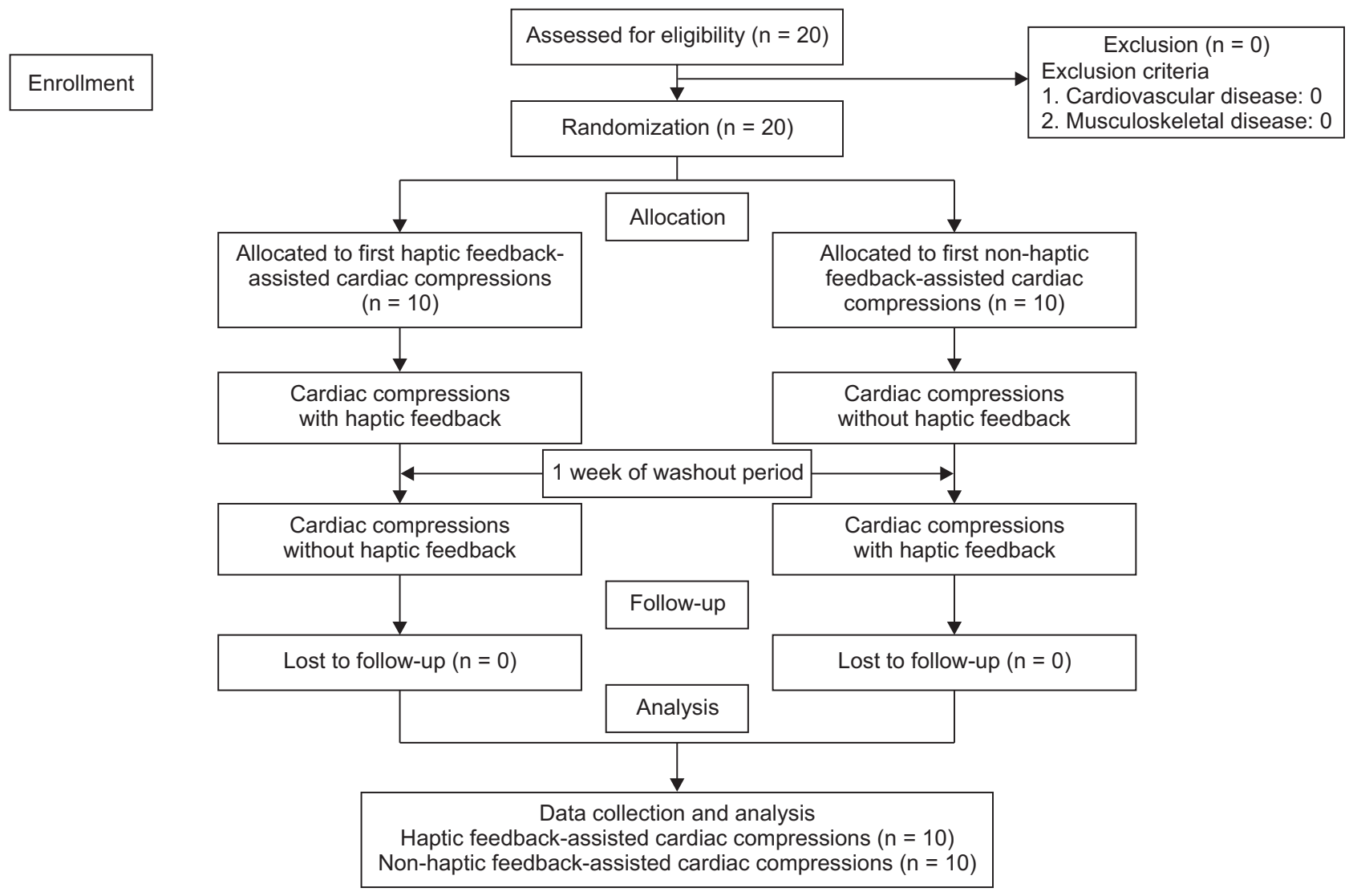

Figure 1. CONSORT flow diagram for the study. 


\section{Study Protocol}

The study was performed with a case-crossover design. On the first visit, the participants were randomly assigned into two groups. The first group performed 2 minutes of continuous CC according to the AHA guidelines with the device (the haptic-first group), and the other group was asked to perform without it (the control-first group). Then, the participants were summoned 1 week later (washout period) to perform CPR as the other group. The study process is illustrated in Figure 1.

\section{Description of the Devices}

An Ambu Man Basic CPR Manikin was used to collect and store the performance data. The R package 'peakPick' was

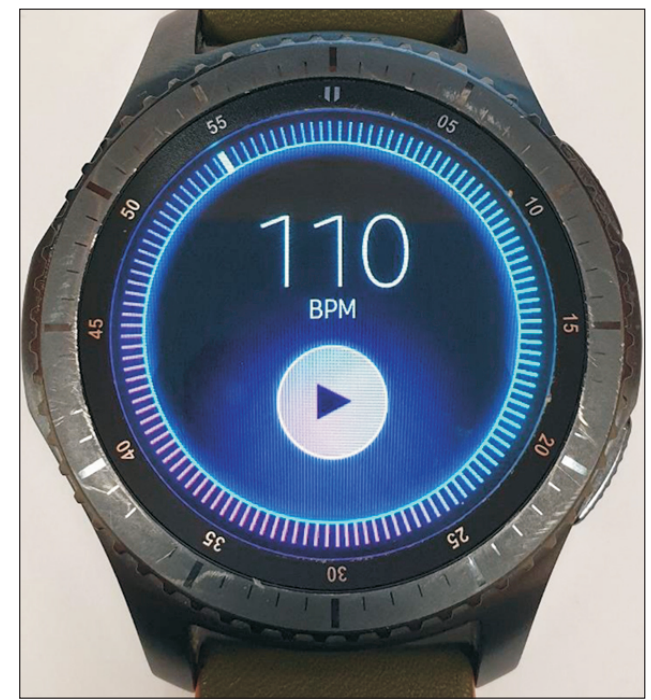

Figure 2. The smartwatch screen displays the rate of $110 / \mathrm{min}$. used to detect peaks and intervals of CCs from the performance data. The haptic device used was a Galaxy Gear S3 frontier smartwatch (Samsung Electronics Inc., Seoul, Korea) with a metronome application (Galaxy Store app Wearable Metronome), which is worn at the wrist to provide information with constant metronome-like vibrations at the rate of $110 / \mathrm{min}$ (Figure 2). Only the haptic feedback system of the metronome application was used in this study.

\section{Measures}

We collected information on demographic factors, such as age, sex, job, and CPR experience. As outcome measures, the total number of CCs was counted during 2 minutes. The participants were assigned to two groups according to their CC performance. The 10 best performing participants without the haptic feedback device were defined as the good performance group (GPG), and the next 10 best performing participants were defined as the bad performance group (BPG). Thirty-second intervals in a sequence were demarcated as quartiles $1 \mathrm{Q}, 2 \mathrm{Q}, 3 \mathrm{Q}$, and $4 \mathrm{Q}$.

\section{Primary Outcome}

The primary outcome was the percentage of the number of adequate rate of CC, defined as $100-120 / \mathrm{min}$, with the haptic feedback device. The total number of CCs was counted during 2 minutes, and between 0.5 and 0.6 seconds for one compression was defined as an adequate duration.

\section{Secondary Outcome}

The secondary outcome was a comparative rate of CC and adequate duration between the good and bad performance

Table 1. Demographic characteristics of the participants

\begin{tabular}{lccc}
\hline & Haptic first group $(\mathbf{n}=10)$ & Control first group $(\mathbf{n}=10)$ & $p$-value \\
\hline Age $(\mathrm{yr})$ & $27.20 \pm 2.69$ & $27.20 \pm 2.69$ & 1.000 \\
Sex, male & $2(10)$ & $3(15)$ & 1.000 \\
BLS or ACLS certification & $10(100)$ & $10(100)$ & 1.000 \\
Experience of cardiac compression & & & \\
Total number & $27.20 \pm 2.69$ & $2.60 \pm 4.95$ & 1.000 \\
Number within 1 month & $2.60 \pm 4.95$ & & 1.000 \\
Job & & $2(10.0)$ & 1.000 \\
EMT & $2(10.0)$ & $8(40.0)$ & $3(15.0)$ \\
Nurse & $8(40.0)$ & $7(35.0)$ \\
Doctor (intern) & $3(15.0)$ & & \\
Doctor (resident) & $7(35.0)$ & & \\
\hline
\end{tabular}

Values are presented as mean \pm standard deviation or number (\%).

BLS: basic life support, ACLS: advanced cardiovascular life support, EMT: emergency medical technician. 
groups; data obtained at each 30-second quartile were analyzed.

\section{Data Analyses}

We described the demographic factors and CPR experience in the two groups. The data were analyzed with the MannWhitney $U$ test, $\chi^{2}$ test, Fisher exact test, or generalized estimation equation (GEE) as appropriate. $p$-values of $<0.05$ were considered statistically significant. The statistical analysis was executed using SAS version 9.4 (SAS Institute, Cary, NC, USA) and R3.5.1. The odds ratio and 95\% confidence intervals (CIs) were estimated from the GEE models that included the main variable and covariates. The primary analysis used to examine the haptic device was the main variant of

Table 2. Results for cardiac compression performance

\begin{tabular}{|c|c|c|c|}
\hline & $\begin{array}{l}\text { Haptic feedback- } \\
\text { assisted CC }\end{array}$ & $\begin{array}{c}\text { Non-haptic } \\
\text { feedback-assisted CC }\end{array}$ & $p$-value \\
\hline \multicolumn{4}{|l|}{ Total } \\
\hline Total number of CCs & 4,209 & 4,224 & \\
\hline Interval of CCs & $0.57 \pm 0.06$ & $0.56 \pm 0.13$ & 0.535 \\
\hline Adequate chest compression number & $2,918(69.33)$ & $2,538(60.09)$ & 0.286 \\
\hline \multicolumn{4}{|l|}{ Subgroup by performance } \\
\hline \multicolumn{4}{|l|}{ Good performance group } \\
\hline Total number of CCs & 2,123 & 2,205 & \\
\hline Interval of CCs & $0.57 \pm 0.06$ & $0.54 \pm 0.03$ & $<0.001$ \\
\hline Adequate chest compression number & $1,597(75.1)$ & $1,951(92.2)$ & $<0.001$ \\
\hline \multicolumn{4}{|l|}{ Bad performance group } \\
\hline Total number of CCs & 2,106 & 2,039 & \\
\hline Interval of CCs & $0.57 \pm 0.07$ & $0.58 \pm 0.18$ & 0.005 \\
\hline Adequate chest compression number & $1,341(63.5)$ & $525(25.4)$ & $<0.001$ \\
\hline \multicolumn{4}{|l|}{ Subgroup by quartiles of duration } \\
\hline \multicolumn{4}{|l|}{$1 \mathrm{Q}$} \\
\hline Total number of CCs & 1,084 & 1,100 & \\
\hline Interval of CCs & $0.56 \pm 0.05$ & $0.55 \pm 0.09$ & 0.016 \\
\hline Adequate chest compression number & $834(76.5)$ & $746(67.2)$ & $<0.001$ \\
\hline \multicolumn{4}{|l|}{$2 \mathrm{Q}$} \\
\hline Total number of CCs & 1,059 & 1,084 & \\
\hline Interval of CCs & $0.57 \pm 0.06$ & $0.55 \pm 0.08$ & $<0.001$ \\
\hline Adequate chest compression number & $749(70.7)$ & $635(58.6)$ & $<0.001$ \\
\hline \multicolumn{4}{|l|}{$3 \mathrm{Q}$} \\
\hline Total number of CCs & 1,045 & 1,064 & \\
\hline Interval of CCs & $0.57 \pm 0.08$ & $0.56 \pm 0.15$ & 0.073 \\
\hline Adequate chest compression number & $719(68.5)$ & $602(56.6)$ & $<0.001$ \\
\hline \multicolumn{4}{|l|}{$4 \mathrm{Q}$} \\
\hline Total number of CCs & 1,038 & 995 & \\
\hline Interval CCs & $0.58 \pm 0.07$ & $0.57 \pm 0.18$ & 0.849 \\
\hline Adequate chest compression number & $637(61.4)$ & $575(57.8)$ & 0.11 \\
\hline
\end{tabular}

Values are presented as mean \pm standard deviation or number (\%).

Comparative rate of CC and adequate duration between the good and bad performance groups; data obtained at each 30-second quartile were analyzed.

CC: cardiac compression. 
interest. We examined the following four models: model 1, unadjusted analysis between the haptic and control groups; model 2, adjusted analysis between the haptic and control groups; model 3, adjusted as model 2 with additional adjustment for the quality group; and model 4 , adjusted as model 3 with additional adjustment for the 4 quartiles.

\section{Results}

Twenty participants were recruited and then randomized into two groups. Two of the participants were paramedics, 8 were nurses, and 10 were physicians. None of the participants was excluded. The demographic characteristics of the participants are summarized in Table 1. No significant differences were found between the two groups regarding sex, age, and experiences.

\section{Primary Outcome}

The CC performance results are shown in Table 2. The mean interval of the haptic and non-haptic feedback-assisted CCs did not show a significant difference, and the adequate chest compression number did not show a significant difference. Figure 3 shows a comparison of the timeline graphs of CCs for each participant and the fitted values of the haptic and non-haptic feedback-assisted CCs. The timeline graphs of CCs for each participant were more consistent with the haptic feedback device.

In the subgroup analysis between the good and bad performance groups, the mean intervals of the haptic and non-

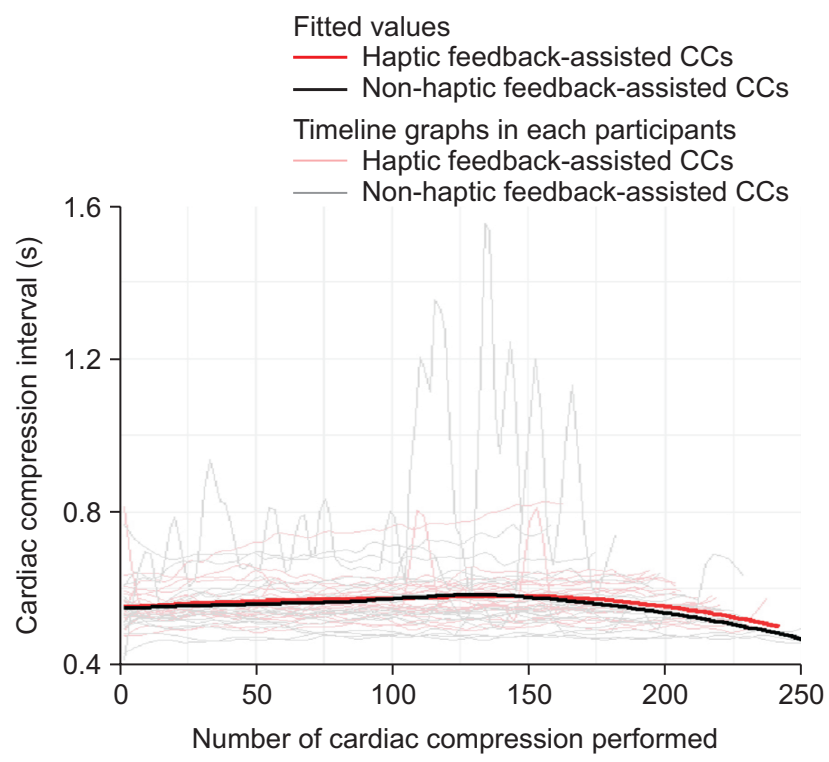

Figure 3. Comparison of the timeline graphs of cardiac compressions (CCs) in each participant and the fitted values of the haptic and non-haptic feedback-assisted CCs. haptic feedback-assisted CCs-good: haptic feedback-assisted (0.57-0.06) vs. non-haptic feedback-assisted (0.54-0.03), $p<0.001$; bad: haptic feedback-assisted, $(0.57-0.07)$ vs. non-haptic feedback assisted (0.58-0.18), $p=0.005$-and the adequate chest compression number showed significant differences-good: haptic feedback assisted (1,597/75.1\%) vs. non-haptic feedback assisted (1,951/92.2\%), $p<0.001$; bad: haptic feedback assisted $(1,341 / 63.5 \%)$ vs. non-haptic feedback assisted (523/25.4\%), $p<0.001$. Figure 4 shows a comparison of the timeline graphs of CCs for each participant and the fitted values of the good and bad performance groups between the haptic and non-haptic feedback-assisted CCs. The fitted values in the bad performance group were more consistent with the haptic feedback device.

In another subgroup analysis, the haptic feedback-assisted CCs showed a generally higher quality from $1 \mathrm{Q}$ to $4 \mathrm{Q}$, but no statistically significant difference was found in $4 \mathrm{Q}$, unlike in $1 \mathrm{Q}$ and $2 \mathrm{Q}$ (Table 2). The mean CC interval and adequate chest compression numbers with and without haptic assistance were the following, respectively: in $1 \mathrm{Q}, 0.56-0.05$ and $0.55-0.09$ ( $p=0.016)$, and $834 / 76 \%$ and $746 / 67.2 \%(p$ $<0.001)$; in 2Q, 0.57-0.06 and 0.55-0.08 $(p<0.001)$, and $749 / 70.7 \%$ and $635 / 58.6 \%(p<0.001)$; in $3 \mathrm{Q}, 0.57-0.08$ and $0.56-0.15(p=0.073)$, and $719 / 68.5 \%$ and $602 / 56.6 \%(p<$ $0.001)$; and in $4 \mathrm{Q}, 0.58-0.07$ and $0.57-0.18(p=0.849)$, and $637 / 61.4 \%$ and $575 / 57.8 \%(p=0.11)$.

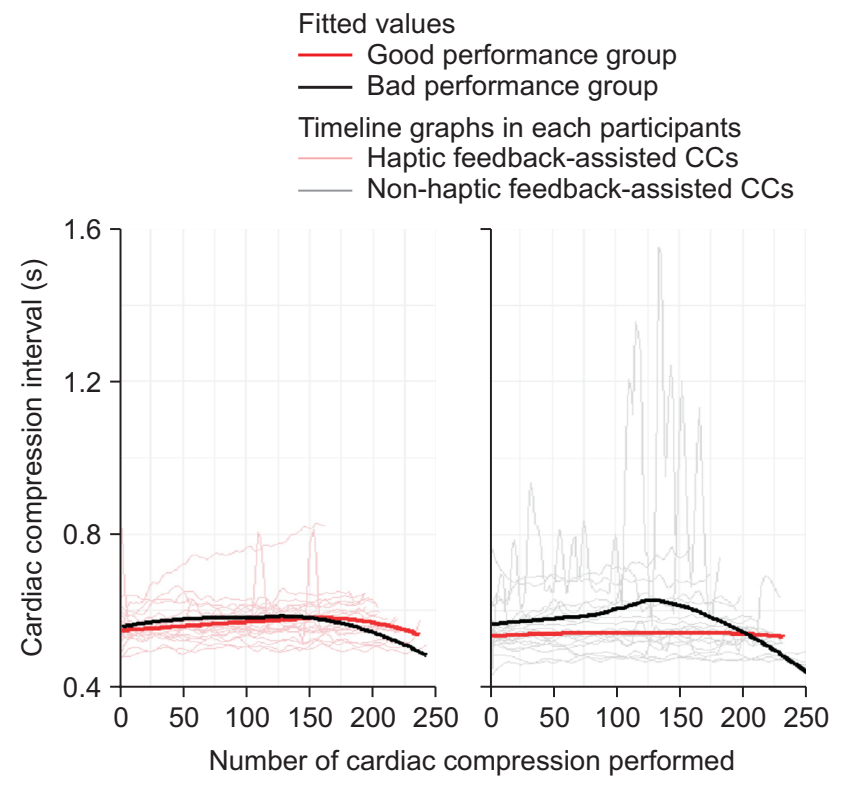

Figure 4. Comparison of the timeline graphs of cardiac compressions (CCs) in each participant and the fitted values of the good and bad performance groups between the haptic and non-haptic feedback-assisted CCs. 


\section{Secondary Outcome}

In the GEE analysis, the unadjusted odds ratio for the haptic feedback device assistance shown in model 1 (Table 3) was 1.502 (95\% CI, 0.712-3.168). Model 2 shows the adjusted odds ratio (AOR) of the quality group and its positive impact. The AORs were 1.71 (95\% CI, 0.69-4.24) and 6.80 (95\% CI, 3.04-15.19) for the GPG and BPG, respectively. The AORs for $2 \mathrm{Q}, 3 \mathrm{Q}$, and $4 \mathrm{Q}$ in comparison with $1 \mathrm{Q}$ were 0.67 (95\% CI, 0.51-0.88), 0.59 (0.41-0.87), and 0.50 (0.32-0.78), respectively. The adjustment for the quality group had a mixed impact on the odds ratio (model 2 vs. model 3) (Table 3). When the haptic feedback device was used, the GPG had a negative impact ( $\mathrm{AOR}=0.25 ; 95 \% \mathrm{CI}, 0.11-0.59)$, but the BPG had $>500 \%$ increase in the likelihood of $\mathrm{CC}$ of adequate duration $(\mathrm{AOR}=5.34 ; 95 \% \mathrm{CI}, 2.16-13.18)$. In the haptic group, the AOR for the GPG in comparison with the BPG was 1.74 (95\% CI, 0.56-5.41). In the control group, the AOR for the GPG in comparison with the BPG was 36.80 (95\%

Table 3. Results from the generalized estimating equation model

\begin{tabular}{|c|c|c|c|c|c|}
\hline & \multirow{2}{*}{ Variable } & \multirow{2}{*}{$\mathrm{OR}$} & \multicolumn{2}{|c|}{$95 \% \mathrm{Cl}$} & \multirow{2}{*}{$p$-value } \\
\hline & & & Lower & Upper & \\
\hline \multirow[t]{2}{*}{ Model 1} & Unadjusted & & & & \\
\hline & Haptic vs. Non-haptic assisted group & 1.502 & 0.712 & 3.168 & 0.286 \\
\hline \multirow[t]{6}{*}{ Model 2} & Adjusted for quality of CC & & & & \\
\hline & Haptic vs. Control & 1.71 & 0.69 & 4.24 & 0.243 \\
\hline & GPG vs. BPG & 6.80 & 3.04 & 15.19 & $<0.0001$ \\
\hline & 2Q vs. $1 \mathrm{Q}$ & 0.67 & 0.51 & 0.88 & 0.004 \\
\hline & 3Q vs. 1Q & 0.59 & 0.41 & 0.87 & 0.007 \\
\hline & $4 \mathrm{Q}$ vs. $1 \mathrm{Q}$ & 0.50 & 0.32 & 0.78 & 0.002 \\
\hline \multirow[t]{8}{*}{ Model 3} & Adjusted for 4 quartiles & & & & \\
\hline & Haptic vs. Control at GPG & 0.25 & 0.11 & 0.59 & 0.002 \\
\hline & Haptic vs. Control at BPG & 5.34 & 2.16 & 13.18 & $<0.0001$ \\
\hline & GPG vs. BPG at haptic & 1.74 & 0.56 & 5.41 & 0.337 \\
\hline & GPG vs. BPG at control & 36.80 & 14.24 & 95.12 & $<0.0001$ \\
\hline & $2 \mathrm{Q}$ vs. $1 \mathrm{Q}$ & 0.63 & 0.47 & 0.85 & 0.003 \\
\hline & 3Q vs. 1Q & 0.55 & 0.37 & 0.83 & 0.004 \\
\hline & $4 \mathrm{Q}$ vs. $1 \mathrm{Q}$ & 0.45 & 0.28 & 0.73 & 0.001 \\
\hline \multirow[t]{12}{*}{ Model 4} & Adjusted for quality of CC during 4 quartiles & & & & \\
\hline & Haptic vs. Control at $1 \mathrm{Q}$ & 1.74 & 0.65 & 4.65 & 0.273 \\
\hline & Haptic vs. Control at 2Q & 1.96 & 0.75 & 5.11 & 0.171 \\
\hline & Haptic vs. Control at 3Q & 1.97 & 0.77 & 5.01 & 0.157 \\
\hline & Haptic vs. Control at 4Q & 1.29 & 0.46 & 3.62 & 0.624 \\
\hline & GPG vs BPG & 6.81 & 3.05 & 15.21 & $<0.0001$ \\
\hline & $2 \mathrm{Q}$ vs. $1 \mathrm{Q}$ at $\mathrm{GPG}$ & 0.71 & 0.50 & 1.02 & 0.063 \\
\hline & $3 \mathrm{Q}$ vs. $1 \mathrm{Q}$ at $\mathrm{GPG}$ & 0.63 & 0.36 & 1.12 & 0.114 \\
\hline & $4 \mathrm{Q}$ vs. $1 \mathrm{Q}$ at $\mathrm{GPG}$ & 0.43 & 0.21 & 0.89 & 0.023 \\
\hline & $2 \mathrm{Q}$ vs. $1 \mathrm{Q}$ at $\mathrm{BPG}$ & 0.63 & 0.40 & 0.99 & 0.044 \\
\hline & $3 \mathrm{Q}$ vs. $1 \mathrm{Q}$ at $\mathrm{BPG}$ & 0.56 & 0.33 & 0.95 & 0.032 \\
\hline & $4 Q$ vs. $1 \mathrm{Q}$ at $\mathrm{BPG}$ & 0.582 & 0.332 & 1.020 & 0.059 \\
\hline
\end{tabular}

Model 1, unadjusted analysis between the haptic and control groups; Model 2, adjusted analysis between the haptic and control groups; Model 3, adjusted as Model 2 with additional adjustment for the quality group; and Model 4, adjusted as Model 3 with additional adjustment for the 4 quartiles; OR: odds ratio, CI: confidence interval, CC: cardiac compression, GPG: good performance group, BPG: bad performance group. 


\section{CI, 14.24-95.12).}

The AORs for $2 \mathrm{Q}, 3 \mathrm{Q}$, and $4 \mathrm{Q}$ in comparison with $1 \mathrm{Q}$ were 0.63 (95\% CI, 0.47-0.85), 0.55 (0.37-0.83), and 0.45 (0.280.73 ), respectively. Moreover, both the quality and quartile were adjusted in model 4 (Table 3). Each quartile had a positive impact when the haptic feedback device was used (1Q: $\mathrm{AOR}=1.74,95 \% \mathrm{CI}, 0.65-4.65 ; 2 \mathrm{Q}: \mathrm{AOR}=1.96,95 \% \mathrm{CI}$, 0.75-5.11; 3Q: AOR $=1.97,95 \% \mathrm{CI}, 0.77-5.01 ; 4 \mathrm{Q}:$ AOR $=1.29,95 \% \mathrm{CI}, 0.46-3.62)$. Both quality groups showed a lower probability of high-quality CC in the time sequence. In the GPG, the AORs for $2 \mathrm{Q}, 3 \mathrm{Q}$, and $4 \mathrm{Q}$ in comparison with 1Q were 0.71 (95\% CI, 0.50-1.02), 0.63 (0.6-1.12), and 0.43 (0.21-0.89), respectively. In the BPG, the AORs for $2 \mathrm{Q}$, $3 \mathrm{Q}$, and $4 \mathrm{Q}$ in comparison with $1 \mathrm{Q}$ were 0.63 (95\% CI, $0.40-$ $0.99), 0.56(0.33-0.95)$, and $0.582(0.332-1.020)$, respectively.

\section{Discussion}

This study demonstrated the capability of a smartwatch using a haptic feedback system for CC. No statistically significant difference in the mean $\mathrm{CC}$ duration or the proportion of CCs of adequate duration was found between the two groups. However, the haptic feedback device-assisted group showed less standard deviation than the non-haptic feedback device-assisted group. The compression rates without CPR feedback devices were significantly faster than the recommended guidelines [23]. In our study, a large number of participants in the non-haptic feedback device-assisted group tended to perform CCs at a faster or slower rate than that recommended in the guidelines or the haptic feedback device-assisted group. This result implies that CPR quality could be homogeneously controlled by using a smartwatch with real-time feedback, and that it is not affected by rescuer experience or the quality of performance.

Previous studies did not show a significant difference in the CC rate in novice rescuers [20,24]. Moreover, even for healthcare professionals, the quality of CPR was suboptimal [25]. In a subgroup analysis, the authors found that the use of the haptic feedback system of a smartwatch significantly increased the proportion of CCs of adequate duration, especially in the poor performance group. To maintain a homogenously good quality of CC during the entire CPR duration, improving the quality $\mathrm{CC}$ in the poor performance group might have great clinical importance.

Recently, the use of smartphones as feedback systems to improve the quality of CC has been introduced in several manikin studies [26]. Rescuers need to grasp the smartphone in the palm when performing CC, which may cause errors in the accelerometers depending on their grasping methods [26]. On the other hand, rescuers usually wear smartwatches on the wrist; therefore, if they wear smartwatch, they might overcome the limitations of smartphones. Thus, smartwatches might be better performing feedback devices than smartphones in real-world situations.

The intensity of vibration of smartwatches might vary according to the manufacturer and cannot be customized. The individual perception of vibration might also vary [27]. Excessive intensity of vibration might interfere with CC performance, and a too weak intensity of vibration might not be perceived. Therefore, further investigation in needed to customize and optimize the intensity of vibration via smartwatches for individual users for better CC performance.

This study had several limitations. First, because this was a simulation study on a manikin, the performance of the compressors would be better than that in real-world clinical practice. The authors assumed the feedback from the watch using vibration would be well delivered even in hectic, noisy environments; however, various features in real-world CPR may interfere with compressor performance.

Second, the AHA guideline recommends a 5- to $6-\mathrm{cm}$ depth and full chest recoil in each compression. The participants were familiar with the guidelines but were reminded again of the guidelines before the study. However, the correct hand position, posture, depth, and chest recoil during CCs were not measured in this study.

Third, the participants in this study were recruited from the emergency department of the study site and were all active healthcare professionals with extensive experience in real-world CPR. Therefore, the performance of compressors might be better than that in real-world. Moreover, the baseline characteristic of the participants, such as their height, weight, or athletic performance were not collected, which may have influenced the quality of CPR.

Finally, this study was conducted for healthcare professionals with hands-only CPR with a compression rate range from 100 to 120 per minute. Therefore, the results might not be applicable in the situation of performing compression to ventilation at a 30:2 ratio.

In conclusion, a CPR feedback system using a smartwatch could not improve rescuers CC performance in terms of the ideal range of CC rate in adherence with the CPR guidelines of the ERC or AHA. In subgroup analysis, the initial compression period of one minute after starting CC might be helpful using a smartwatch to increase the percentage of adequate compression. 


\section{Conflict of Interest}

No potential conflict of interest relevant to this article was reported.

\section{ORCID}

Boram Choi (https://orcid.org/0000-0001-9244-604X)

Taerim Kim (https://orcid.org/0000-0002-8149-1205)

Sun Young Yoon (https://orcid.org/0000-0002-4320-2388)

Jun Sang Yoo (https://orcid.org/0000-0003-3696-3813)

Ho-Jeong Won (https://orcid.org/0000-0001-8196-0879)

Kyunga Kim (https://orcid.org/0000-0002-0865-2236)

Eun Jin Kang (https://orcid.org/0000-0002-6324-1448)

Hee Yoon (https://orcid.org/0000-0002-1297-9813)

Sung Yeon Hwang (https://orcid.org/0000-0002-1352-3009)

Tae Gun Shin (https://orcid.org/0000-0001-9657-1040)

Min Seob Sim (https://orcid.org/0000-0001-6645-3684)

Won Chul Cha (https://orcid.org/0000-0002-2778-2992)

\section{References}

1. Abella BS, Alvarado JP, Myklebust H, Edelson DP, Barry A, O'Hearn N, et al. Quality of cardiopulmonary resuscitation during in-hospital cardiac arrest. JAMA 2005;293(3):305-10.

2. Eisenberg MS, Mengert TJ. Cardiac resuscitation. N Engl J Med 2001;344(17):1304-13.

3. Rea TD, Crouthamel M, Eisenberg MS, Becker LJ, Lima AR. Temporal patterns in long-term survival after resuscitation from out-of-hospital cardiac arrest. Circulation 2003;108(10):1196-201.

4. Daya MR, Schmicker RH, Zive DM, Rea TD, Nichol G, Buick JE, et al. Out-of-hospital cardiac arrest survival improving over time: Results from the Resuscitation Outcomes Consortium (ROC). Resuscitation 2015;91:108-15.

5. Feneley MP, Maier GW, Kern KB, Gaynor JW, Gall SA $\mathrm{Jr}$, Sanders AB, et al. Influence of compression rate on initial success of resuscitation and 24 hour survival after prolonged manual cardiopulmonary resuscitation in dogs. Circulation 1988;77:240-50.

6. Meaney PA, Bobrow BJ, Mancini ME, Christenson J, de Caen AR, Bhanji F, et al. Cardiopulmonary resuscitation quality: [corrected] improving cardiac resuscitation outcomes both inside and outside the hospital: a consensus statement from the American Heart Association. Circulation 2013;128(4):417-35.
7. Kleinman ME, Brennan EE, Goldberger ZD, Swor RA, Terry M, Bobrow BJ, et al. Part 5: Adult basic life support and cardiopulmonary resuscitation quality: 2015 American Heart Association Guidelines Update for Cardiopulmonary Resuscitation and Emergency Cardiovascular Care. Circulation 2015;132(18 Suppl 2):S414-35.

8. Link MS, Berkow LC, Kudenchuk PJ, Halperin HR, Hess EP, Moitra VK, et al. Part 7: Adult advanced cardiovascular life support: 2015 American Heart Association Guidelines Update for Cardiopulmonary Resuscitation and Emergency Cardiovascular Care. Circulation 2015;132(18 Suppl 2):S444-64.

9. Perkins GD, Olasveengen TM, Maconochie I, Soar J, Wyllie J, Greif R, et al. European Resuscitation Council Guidelines for Resuscitation: 2017 update. Resuscitation 2018;123:43-50.

10. Wik L, Kramer-Johansen J, Myklebust H, Sorebo H, Svensson L, Fellows B, et al. Quality of cardiopulmonary resuscitation during out-of-hospital cardiac arrest. JAMA 2005;293(3):299-304.

11. Kirkbright S, Finn J, Tohira H, Bremner A, Jacobs I, Celenza A. Audiovisual feedback device use by health care professionals during CPR: a systematic review and meta-analysis of randomised and non-randomised trials. Resuscitation 2014;85(4):460-71.

12. Martin P, Theobald P, Kemp A, Maguire S, Maconochie I, Jones M. Real-time feedback can improve infant manikin cardiopulmonary resuscitation by up to $79 \%$ : a randomised controlled trial. Resuscitation 2013;84(8):112530.

13. Oh J, Song Y, Kang B, Kang H, Lim T, Suh Y, et al. The use of dual accelerometers improves measurement of chest compression depth. Resuscitation 2012;83(4):5004.

14. Yu BG, Oh JH, Kim Y, Kim TW. Accurate measurement of chest compression depth using impulseradio ultra-wideband sensor on a mattress. PLoS One 2017;12(8):e0183971.

15. Bhanji F, Donoghue AJ, Wolff MS, Flores GE, Halamek LP, Berman JM, et al. Part 14: Education: 2015 American Heart Association Guidelines Update for Cardiopulmonary Resuscitation and Emergency Cardiovascular Care. Circulation 2015;132(18 Suppl 2):S561-73.

16. Greif R, Lockey AS, Conaghan P, Lippert A, De Vries W, Monsieurs KG, et al. European Resuscitation Council Guidelines for Resuscitation 2015: Section 10. Education and implementation of resuscitation. Resuscitation 2015;95:288-301. 
17. Chen KY, Ko YC, Hsieh MJ, Chiang WC, Ma MH. Interventions to improve the quality of bystander cardiopulmonary resuscitation: a systematic review. PLoS One 2019;14(2):e0211792.

18. Abella BS, Edelson DP, Kim S, Retzer E, Myklebust H, Barry AM, et al. CPR quality improvement during inhospital cardiac arrest using a real-time audiovisual feedback system. Resuscitation 2007;73(1):54-61.

19. Gruenerbl A, Pirkl G, Monger E, Gobbi M, Lukowicz P. Smart-watch life saver: smart-watch interactive-feedback system for improving bystander CPR. Proceedings of the 2015 ACM International Symposium on Wearable Computers; 2015 Sep 7-11; Osaka, Japan. p. 19-26.

20. Ahn C, Lee J, Oh J, Song Y, Chee Y, Lim TH, et al. Effectiveness of feedback with a smartwatch for highquality chest compressions during adult cardiac arrest: a randomized controlled simulation study. PLoS One 2017;12(4):e0169046.

21. Song Y, Chee Y, Oh J, Ahn C, Lim TH. Smartwatches as chest compression feedback devices: a feasibility study. Resuscitation 2016;103:20-3.

22. Reeder B, David A. Health at hand: a systematic review of smart watch uses for health and wellness. J Biomed
Inform 2016;63:269-76.

23. Lu TC, Chang YT, Ho TW, Chen Y, Lee YT, Wang YS, et al. Using a smartwatch with real-time feedback improves the delivery of high-quality cardiopulmonary resuscitation by healthcare professionals. Resuscitation 2019;140:16-22.

24. Lee J, Song Y, Oh J, Chee Y, Ahn C, Shin H, et al. Smartwatch feedback device for high-quality chest compressions by a single rescuer during infant cardiac arrest: a randomized, controlled simulation study. Eur J Emerg Med 2019;26(4):266-71.

25. Abella BS, Sandbo N, Vassilatos P, Alvarado JP, O'Hearn $\mathrm{N}$, Wigder $\mathrm{HN}$, et al. Chest compression rates during cardiopulmonary resuscitation are suboptimal: a prospective study during in-hospital cardiac arrest. Circulation 2005;111(4):428-34.

26. Song Y, Oh J, Chee Y. A new chest compression depth feedback algorithm for high-quality CPR based on smartphone. Telemed J E Health 2015;21(1):36-41.

27. Lundstrom R, Stromberg T, Lundborg G. Vibrotactile perception threshold measurements for diagnosis of sensory neuropathy: description of a reference population. Int Arch Occup Environ Health 1992;64(3):201-7. 